\title{
Autophagic tumor-associated macrophages promote the endothelial mesenchymal transition in lung adenocarcinomas through the FUT4/p-ezrin pathway
}

\author{
Kangwu Wang ${ }^{1}$, Xiao Chen ${ }^{2}$ \\ ${ }^{1}$ Department of Thoracic Surgery, The First Affiliated Hospital of Bengbu Medical College, Bengbu, China; ${ }^{2}$ Department of Geriatrics, The First \\ Affiliated Hospital of Bengbu Medical College, Bengbu, China \\ Contributions: (I) Conception and design: Both authors; (II) Administrative support: X Chen; (III) Provision of study materials or patients: K Wang; \\ (IV) Collection and assembly of data: K Wang; (V) Data analysis and interpretation: Both authors; (VI) Manuscript writing: Both authors; (VII) Final \\ approval of manuscript: Both authors. \\ Correspondence to: Dr. Xiao Chen. Department of Geriatrics, The First Affiliated Hospital of Bengbu Medical College, 287 Changhuai Road, Bengbu \\ 233000, China. Email: chenxiao0552@163.com.
}

Background: Lung adenocarcinoma is one of the most common malignant tumors with high morbidity and mortality, but the effect of Tumor-associated macrophages (TAMs) on lung adenocarcinoma has not been studied clearly now.

Methods: In this study, TAMs were stably transfected with Atg5 silence or overexpression lentiviral vectors to inhibit or induce autophagy of TAMs. In addition, the expression of fucosyltransferase IV (FUT4) or Ezrin were interfered in TAMs with autophagy. The above treated TAMs were then cocultured with A549 or H1299 cells. The expressions of genes were detected by qPCR, western blotting, cell immunofluorescence, and enzyme-linked immunosorbent assay. Meanwhile, cell migration and invasion were analyzed by Transwell assay and wound healing assay. Furthermore, the effects of TAMs with autophagy were explored in lung adenocarcinoma xenograft model of mice.

Results: The results showed that overexpression of autophagy-related gene 5 (ATG5) induced autophagy in TAMs, which increased the expression of FUT4, TGF- $\beta 1$, and p-ezrin, and promoted epithelialmesenchymal transition (EMT) in lung adenocarcinoma cells. However, FUT4 silencing partially reversed the effects of TAM autophagy, specifically, the expression of TGF- $\beta 1$ and p-ezrin was inhibited and EMT in lung adenocarcinoma cells was suppressed. Notably, ezrin deletion in autophagic TAMs induced by rapamycin reduced TGF- $\beta 1$ expression and suppressed EMT in lung adenocarcinoma cells. Consistently, in vivo experiments also revealed that autophagic TAMs increased the expression of FUT4, TGF- $\beta 1$, and p-ezrin, and promoted EMT in lung adenocarcinomas. Similarly, FUT4 silencing partially reversed the effects of autophagic TAMs on EMT in lung adenocarcinomas.

Conclusions: In conclusion, autophagic TAMs promoted TGF- $\beta 1$ secretion through the FUT4/p-ezrin pathway and induced EMT in co-cultured lung adenocarcinoma cells.

Keywords: Tumor-associated macrophages (TAMs); autophagy; epithelial-mesenchymal transition (EMT); lung adenocarcinoma; fucosyltransferase IV

Submitted Aug 22, 2021. Accepted for publication Oct 16, 2021.

doi: $10.21037 /$ jtd-21-1519

View this article at: https://dx.doi.org/10.21037/jtd-21-1519 


\section{Introduction}

Lung adenocarcinoma is one of the most common malignant tumors and is associated with high morbidity and mortality (1). Currently, the conventional treatments for patients with lung adenocarcinoma include surgical resection, chemotherapy, and personalized pharmacological treatments (2). Despite this, patient prognosis can be hampered by drug resistance, metastasis, and recurrence $(3,4)$. Therefore, understanding the underlying mechanisms of metastasis and recurrence is crucial for developing effective treatments for patients with lung adenocarcinoma.

Recently, increasing evidence has demonstrated the pivotal role of the tumor microenvironment (TME) in regulating malignant biological properties (5). The TME consists of various stromal cells (such as macrophages, fibroblasts, and endothelial cells), tumor cells, cytokines, and chemokines (6). The stromal cells in the TEM play an important role in promoting the formation of tumor invasion and metastasis (7). Notably, tumor-associated macrophages (TAMs) are infiltrating macrophages in tumor tissues that account for the largest proportion of tumor stromal cells and the most abundant cell type in the TME (8). Previous studies have suggested that TAMs are involved in promoting tumor growth and metastasis, as well as inducing tumor angiogenesis and immunosuppression (9-11). Report has found that M1 macrophages inhibit tumor growth, whereas M2 macrophages promote tumor growth and metastasis in lung adenocarcinoma (12). Wang et al. found that M2 macrophages promoted FUT4/LeY expression in lung adenocarcinoma cells, and FUT4/LeY promoted the phosphorylation of Ezrin, which was the critical mechanism of M2 macrophages-induced epithelialmesenchymal transition (EMT) (13).

Autophagy is considered a protective mechanism to maintain cellular homeostasis (14). Autophagy provides basic substances and energy for cell recycling by degrading intracellular proteins and organelles, thereby promoting cell adaptation to environmental changes and enhancing cell survival ability $(14,15)$. However, autophagy induced by a persistent stressed state will result in cell apoptosis $(14,15)$. Autophagy has a dynamic role in malignancies as both a tumor-promoter and a tumor-suppressor depending on the different stages of cancer development (16). The mammalian target of rapamycin (mTOR) signaling pathway has been shown to be closely associated with autophagy, and activated mTOR can suppress the occurrence of autophagy (17). A previous study showed that mTOR inhibitors, such as rapamycin, can contribute to cell survival by enhancing autophagy in lung adenocarcinoma cells (18).

Interestingly, autophagy mediated by TAMs can promote angiogenesis and EMT in tumors through autocrine or paracrine mediated interactions, and further activate inflammatory cells, disrupt the cytokine network, and escape immune surveillance, all of which contribute to the initiation and progression of tumors $(9,19)$. In addition, it has been reported that TMEs modulate EMT of human adenocarcinoma cells in a vitro $3 \mathrm{D}$ cancer model (20). However, few studies have investigated the effects of autophagic TAM in the development of lung adenocarcinoma and the mechanisms involved. In the present study, we cultured THP-1-derived macrophages as TAMs and autophagy was induced in TAMs by upregulating the expression of autophagy related gene 5 (ATG5) or by treatment with rapamycin. The association between autophagic TAM and EMT was examined in vitro by co-culture of TAMs with lung adenocarcinoma cells, and in vivo using a mouse tumor xenograft model. We present the following article in accordance with the ARRIVE reporting checklist (available at https://dx.doi.org/10.21037/ jtd-21-1519).

\section{Methods}

\section{Cell culture}

The human leukemia monocytic cell line THP-1 and the human lung adenocarcinoma cell lines A549 and H1299 were obtained from Nanjing KeyGen Biotech. THP-1 cells were cultured in complete 1640 medium (Thermo, China) at $37^{\circ} \mathrm{C}$ in a $5 \%$ carbon dioxide atmosphere.

\section{Polarization of TAMs in vitro}

THP-1 cells were treated with $25 \mathrm{ng} / \mathrm{mL}$ phorbol 12-myristate 13-acetate (PMA, Sigma) for 48 hours to induce cell differentiation into macrophages (M0). The macrophages were then exposed to $20 \mathrm{ng} / \mathrm{mL}$ interleukin-4 (IL-4, Sigma) for 48 hours to obtain M2-like TAM polarization. Flow cytometry analysis was performed to detect the expression of macrophage surface markers on the TAMs.

\section{Cell treatment and transfection}

ATG5 silencing and overexpression lentiviral vectors, fucosyltransferase IV (FUT4) silencing and overexpression 
Table 1 Specific primers for qPCR assay

\begin{tabular}{ll}
\hline Gene & Primer sequence \\
\hline Atg5 & Sense primer: 5'-TCAGCCACTGCAGAGGTGTTT-3' \\
& Antisense primer: 5'-GGCTGCAGATGGACAGTTGCA-3' \\
FUT4 & Sense primer: 5'-TCCTACGGAGAGGCTCAG-3' \\
& Antisense primer: 5'-TCCTCGTAGTCCAACACG-3' \\
TGF- $\beta 1$ & Sense primer: 5'-CTAATGGTGGACCGCAACAACG-3' \\
& Antisense primer: 5'-CCTGTATTCCGTCTCCTTGG-3' \\
Ezrin & Sense primer: 5'-TGGAGTTGATGCCCTTGGAC-3' \\
& Antisense primer: 5'-AGTCAGGTGCCTTCTTGTCG-3' \\
GAPDH & Sense primer: 5'-GTGGATCAGCAAGCAGGAGT-3' \\
& Antisense primer: 5'-AAAGCCATGCCAATCTCATC-3'
\end{tabular}

plasmids, ezrin silencing plasmid, and their corresponding negative controls were obtained from GenePharma (Shanghai, China). The TAMs were stably transfected with ATG5 silencing or overexpression lentiviral vectors to establish stably silenced ATG5 TAMs (shATG5-TAMs: 5'-AAA CAA GUU GGA AUU CGU CCA-3') and stably overexpressed ATG5 TAMs (ATG5-TAMs), respectively. The respective control TAMs were designated scramble-TAMs and vector-TAMs. To investigate the effects of TAM-induced autophagy on human lung adenocarcinoma cells, shATG5-TAMs and ATG5-TAMs were co-cultured with A549 and H1299 cells.

To evaluate the role of FUT4 in TAM-mediated autophagy in lung adenocarcinoma, FUT4 silencing and overexpression plasmids were transfected into ATG5-TAMs (shFUT4-TAMs: 5'-AGU UGA AGA GGU UAC UUG CCA-3') and shATG5-TAMs using the Lipofectamine ${ }^{\mathrm{TM}}$ 3000 transfection reagent (Thermo), and co-cultured with H1299 cells and A549 cells in the presence of rapamycin.

To investigate the role of Ezrin in TAM-mediated autophagy in lung adenocarcinoma, TAMs were transfected with the Ezrin silencing plasmid (sh-Ezrin: 5'-AAU CUU CAG GUA UUC CAA CAU-3'), and co-cultured with H1299 cells in the presence of rapamycin.

\section{Animal experiments}

A total of 20 four-week-old male BALB/c Nude mice $(18-20 \mathrm{~g})$ (Charles river, Beijing, China) were housed in our laboratory. To establish the tumor xenograft model, $1 \times 10^{7}$ H1299 cells were subcutaneously injected into the right flank of the nude mice. The tumor xenograft mice $(\mathrm{n}=5$ in each group) were then exposed to saline (H1299 group), TAMs (TAMs + H1299 group), ATG5-shNC-TAMs (ATG5-shNC-TAMs + H1299 group), or ATG5-shFUT4TAMs (ATG5-shFUT4-TAMs + H1299 group) via direct injection into the tumor. Tumor volumes were measured every 3 days for 30 days. Experiments were performed under a project license (No. 2020-146) granted by the ethics committee of the First Affiliated Hospital of Bengbu Medical College, in compliance with China national or institutional guidelines for the care and use of animals.

\section{Quantitative reverse transcription polymerase chain reaction (RT-PCR)}

The treated cells and tumor tissues were collected and total RNA was extracted using Invitrogen TRIzol Reagent (Thermo). Complementary DNA samples were prepared using the QuantiTect Reverse Transcription Kit (QIAGEN). Quantitative PCR was performed using the SYBR Premix Ex Taq TM II kit (Takara). Glyceraldehyde 3-phosphate dehydrogenase (GAPDH) was used as the internal standard for mRNA quantitation. The sequences of the primers used are presented in Table 1.

\section{Western blot analysis}

Total protein was extracted from the treated cells and tumor tissues using ice-cold lysis buffer (Beyotime, China). Proteins samples were then resolved by polyacrylamide gel electrophoresis (PAGE) and transferred to polyvinylidene fluoride (PVDF) membranes. Membranes were then blocked, followed by probing with primary antibodies against ATG5 (ab108327, Abcam), microtubule-associated protein 1A/1Blight chain 3 (LC3; ab51520, Abcam), beclin-1 (ab207612, Abcam), p62 (ab109012, Abcam), FUT4 (ab231561, Abcam), p-ezrin (ab76247, Abcam), tumor growth factor (TGF)- $\beta 1$ (ab215715, Abcam), N-cadherin (N-cad; ab18203, Abcam), E-cadherin (E-cad; ab40772, Abcam), vimentin (ab92547, Abcam), Snail (ab216347, Abcam), Slug (ab106077, Abcam), or GAPDH (ab181602, Abcam) at room temperature for 3 hours. Membranes were then washed, followed by incubation with the second antibody (ab6721, Abcam). Protein expression was observed using enhanced chemiluminescence (ECL, Millipore, USA).

\section{Cell immunofluorescence}

Cells were fixed onto slides using $4 \%$ formaldehyde solution 
for 12 minutes at room temperature, washed with phosphate buffered saline (PBS) solution three times, and blocked with $5 \%$ bovine serum albumin (BSA) solution. Slides were then incubated with antibodies targeting LC3 (ab243506, Abcam), FUT4 (ab212396, Abcam), and p-ezrin (ab76247, Abcam) overnight at $4{ }^{\circ} \mathrm{C}$. After washing in PBS solution, slides were incubated in the dark with the secondary antibodies (goat anti-rabbit IgG H\&L (FITC), ab6717, Abcam; goat polyclonal secondary antibody to mouse IgGH\&L (Alexa Fluor ${ }^{\circledR}$ 647), ab150115, Abcam) for 1-2 hours. The cell nuclei stained with 4',6-diamidino-2-phenylindole (DAPI) and slides were mounted with the Prolong Gold Antifade Reagent (CST). The fluorescence intensities were evaluated by observation under fluorescence microscopy.

\section{Enzyme-linked immunosorbent assay (ELISA)}

The relative content of TGF- $\beta 1$ in the treated cells and tumor tissues were detected using the TGF- $\beta 1$ ELISA kit (Solarbio Life Science Company (Beijing, China) in accordance with the manufacturer's instructions.

\section{Transwell assay}

Briefly, A549 and H1299 cells were seeded in RPMI 1640 media without serum into the top compartment of the transwell. Complete RPMI 1640 culture medium was added to the lower compartment as the chemotactic factor and the chambers were incubated at $37^{\circ} \mathrm{C}$. A cotton swab was used to remove the noninvasive cells. Cells in the lower compartment were stained with crystal violet and the migrated and invasive cells were counted under a light microscope.

\section{Wound healing assay}

The treated A549 and H1299 cells were cultured in 6-well plates to $60-70 \%$ confluency. A sterile pipette tip was used to scratch the cells in a vertical lineation. Cells were then cultured in DMEM without serum. The width of the scratch was assessed by light microscopy at 1 hour and 24 hours, and the cell migration rate was calculated.

\section{Statistical analysis}

All experiments were repeated technically three times. Statistical analyses were conducted using SPSS 20.0 statistical software. Differences between sample groups and within each sample group were analyzed by analysis of variance (ANOVA) or by independent-samples $t$-test. Quantitative values are expressed as the mean \pm standard deviation (SD) and $\mathrm{P}$ value less than 0.05 was considered statistically significant in all experiments.

\section{Results}

\section{Autophagic TAMs promotes endothelial mesenchymal transition (EMT) in co-cultured lung adenocarcinoma cells}

To investigate the effects of TAM autophagy in cocultured lung adenocarcinoma cells, autophagy was inhibited by ATG5 silencing (shATG5-TAMs) or promoted via ATG5 overexpression (ATG5-TAMs). Compared with control cells (scramble-TAMs and vectorTAMs), the mRNA and protein levels of ATG5 were significantly decreased in shATG5-TAMs and significantly increased in ATG5-TAMs (Figure 1A,1B). The expression of autophagy-associated proteins LC3, beclin-1, and p62 were assessed by Western blot and immunofluorescence analysis. In the shAtg5-TAMs, the expression of LC3 II/I and beclin-1 were down-regulated while the expression of p62 was up-regulated. Conversely, ATG5-TAMs showed upregulated LC3 II/I and beclin-1 and down-regulated expression of p62 (Figure 1B,1C). These results suggested that the promotion or inhibition of autophagy in TAMs was successfully induced. Furthermore, ATG5 silencing inhibited the expression of FUT4, p-ezrin and TGF- $\beta 1$, while ATG5 overexpression promoted their expression (Figure $1 A-1 D)$. Since TGF- $\beta 1$ can induce cancer cell EMT, the results suggested that TAMs autophagy may lead to EMT in lung adenocarcinoma cells, and FUT4 and p-ezrin may participate in this process.

To verify the above hypothesis, shATG5-TAMs and ATG5-TAMs were co-cultured with A549 and H1299 cells. Co-cultures of scramble-TAMs with A549 cells and vector-TAMs with H1299 cells were used as controls. The expression of EMT biomarkers including N-cad, E-cad, vimentin, Snail, and Slug were detected by Western blot. The results showed that compared to A549 cells or H1299 cells alone, co-culture with normal TAMs resulted in downregulation of E-cad and up-regulation of $\mathrm{N}$-cad, vimentin, Snail, and Slug. However, co-culture with shATG5-TAMs elevated the expression of E-cad and reduced the expression of N-cad, vimentin, Snail, and Slug in A549 cells. Conversely, co-culture with ATG5-TAMs exhibited the opposite effect in H1299 cells, namely, reduced expression of E-cad and increased expression of N-cad, vimentin, Snail, and Slug 
A

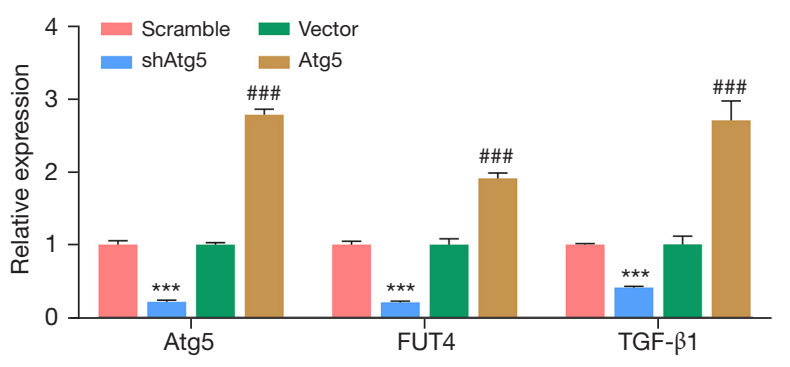

C

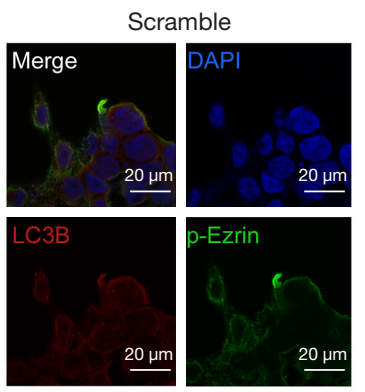

B
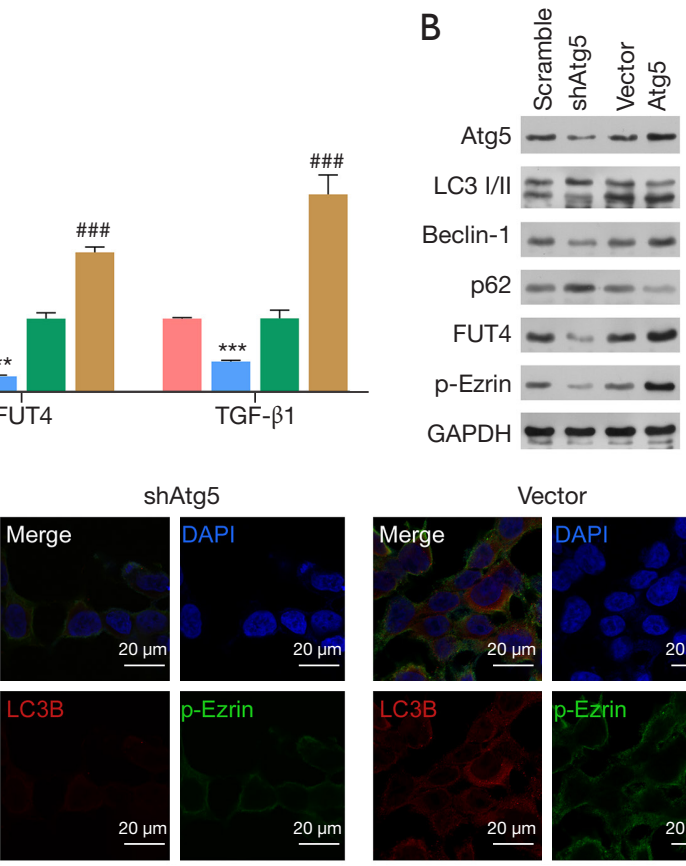

$\operatorname{shAtg} 5$

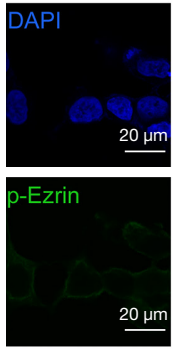

Vector

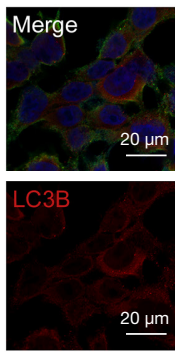

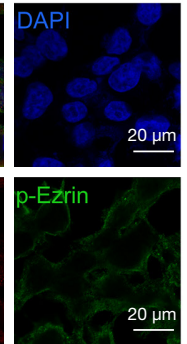
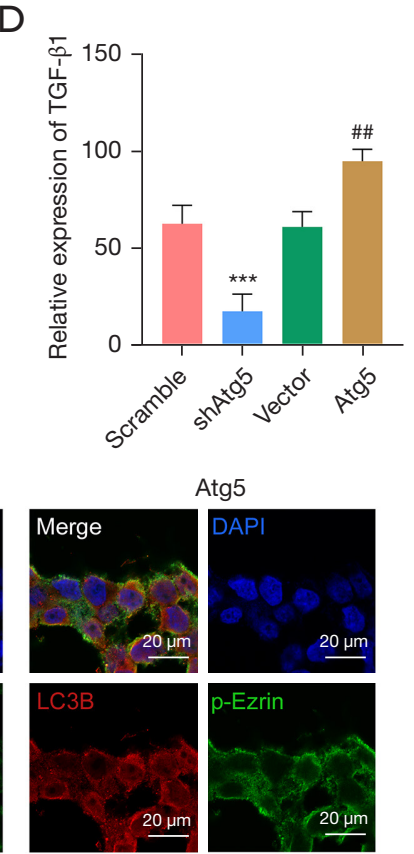

Figure 1 The role of FUT4, p-ezrin, and TGF- $\beta 1$ in TAM autophagy. (A) The mRNA levels of ATG5, FUT4, and TGF- $\beta 1$ in shATG5TAMs, scramble-TAMs, ATG5-TAMs, and vector-TAMs by qRT-PCR; (B) the protein levels of ATG5, LC3, beclin-1, p62, FUT4, and p-ezrin in shATG5-TAMs, scramble-TAMs, ATG5-TAMs, and vector-TAMs by Western blotting; (C) cell immunofluorescence showing the expression of LC3 and p-ezrin in shATG5-TAMs, scramble-TAMs, ATG5-TAMs, and vector-TAMs; (D) the expression of TGF- $\beta 1$ in shATG5-TAMs, scramble-TAMs, ATG5-TAMs, and vector-TAMs assessed by enzyme-linked immunosorbent assay. ${ }^{* * *}$,

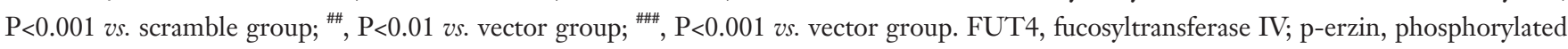
erzin; TGF- $\beta 1$, tumor growth factor $\beta 1$; TAM, tumor-associated macrophage; ATG5, autophagy related gene 5 ; sh, short hairpin; qRTPCR, quantitative reverse transcription polymerase chain reaction; LC3, microtubule-associated protein 1A/1B-light chain 3; GAPDH, glyceraldehyde 3-phosphate dehydrogenase.

(Figure 2A). These results suggested that co-culturing lung adenocarcinoma cells with TAMs could induced EMT and TAM-induced autophagy may play a vital role in this process.

The cell migration and invasion ability of the lung adenocarcinoma cells were evaluated using the wound healing assay and the transwell assay. Compared with A549 cells or H1299 cells that were cultured alone, co-culture of normal TAMs with A549 cells or H1299 cells showed increased relative wound area, as well as increased rates of cell migration and cell invasion (Figure 2B,2C). Notably, both the relative wound area, as well as the cell migration and invasion rates were remarkably decreased in A549 cells that were co-cultured with shATG5-TAMs. Conversely, ATG5-TAMs promoted cell migration and invasion of H1299 cells (Figure 2B,2C). These results suggested that TAM-mediated autophagy can induce the migration and invasion ability of lung adenocarcinoma cells and this process is facilitated by ATG5 expression.

\section{The effects of FUT4 silencing on the EMT of human lung adenocarcinoma cells induced by autophagic TAMs}

To further explore the role of FUT4 expression in the EMT of H1299 cells induced by TAM autophagy, experiments were conducted to alter the FUT4 expression in ATG5-TAMs. The silencing of FUT4 was confirmed by the lower expression of FUT4 in ATG5-shFUT4-TAMs compared to the negative control ATG5-shNC-TAMs (Figure 3A-3C). FUT4 silencing in TAMs inhibited the expression of p-ezrin and TGF- $\beta 1$, without changing the expression of LC3 (Figure $3 A-3 D$ ), suggesting that FUT4 silencing reversed the inhibitory effects of TAM autophagy on the expression of p-ezrin and TGF- $\beta 1$. Furthermore, ATG5-shFUT4-TAMs and ATG5-shNC TAMs were stimulated with rapamycin to enhance autophagy and 
A

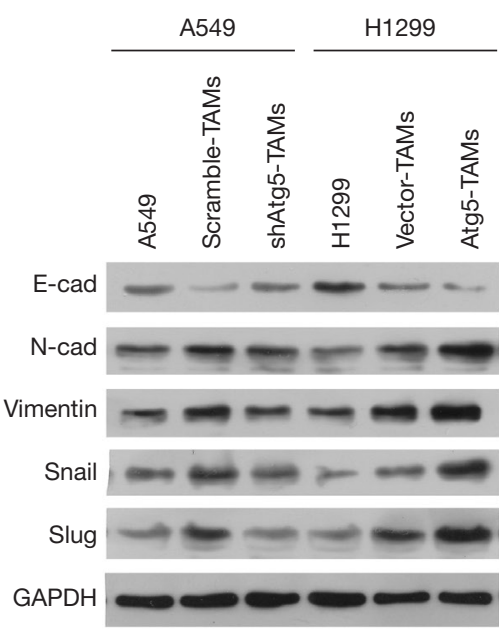

B
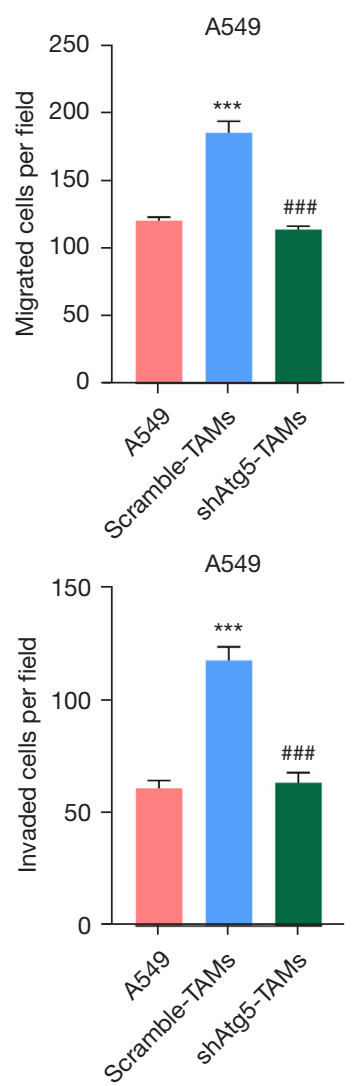

C

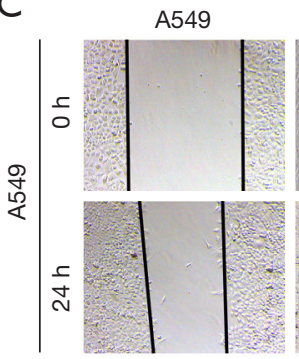

Scamble-TAMs
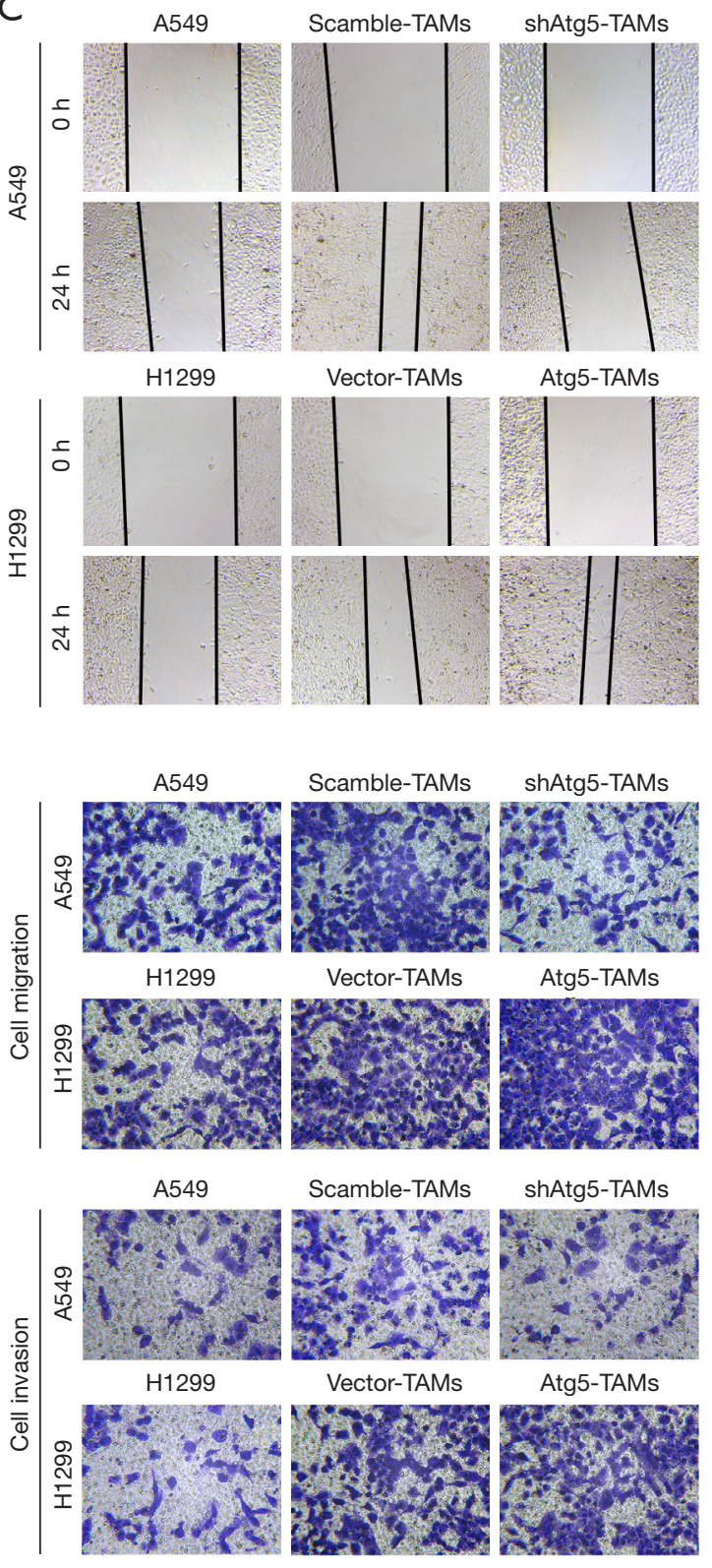

Figure 2 TAM autophagy induced EMT of human lung adenocarcinoma cells. Autophagy-induced (ATG5-TAMs) and autophagyinhibited (shATG5-TAMs) TAMs and were co-cultured with A549 and H1299 cells. (A) Western blot analysis showing the protein expression of N-cadherin (N-cad), E-cadherin (E-cad), vimentin, Snail, and Slug in the co-cultured A549 and H1299 cells; (B) Transwell assays showing the cell migration and invasion ability of the co-cultured A549 and H1299 cells (×100 magnification), in which cells in the lower compartment were stained with crystal violet; (C) wound healing assays showing the relative wound area of the co-cultured A549 and H1299 cells (×40 magnification). ${ }^{* * *}, \mathrm{P}<0.001$ vs. A549 or H1299 group; ${ }^{\# \# \#, ~} \mathrm{P}<0.001$ vs. scramble + TAMs group. TAM, tumor-associated macrophage; ATG5, autophagy related gene 5; sh, short hairpin; EMT, endothelial mesenchymal transition; GAPDH, glyceraldehyde 3-phosphate dehydrogenase. 

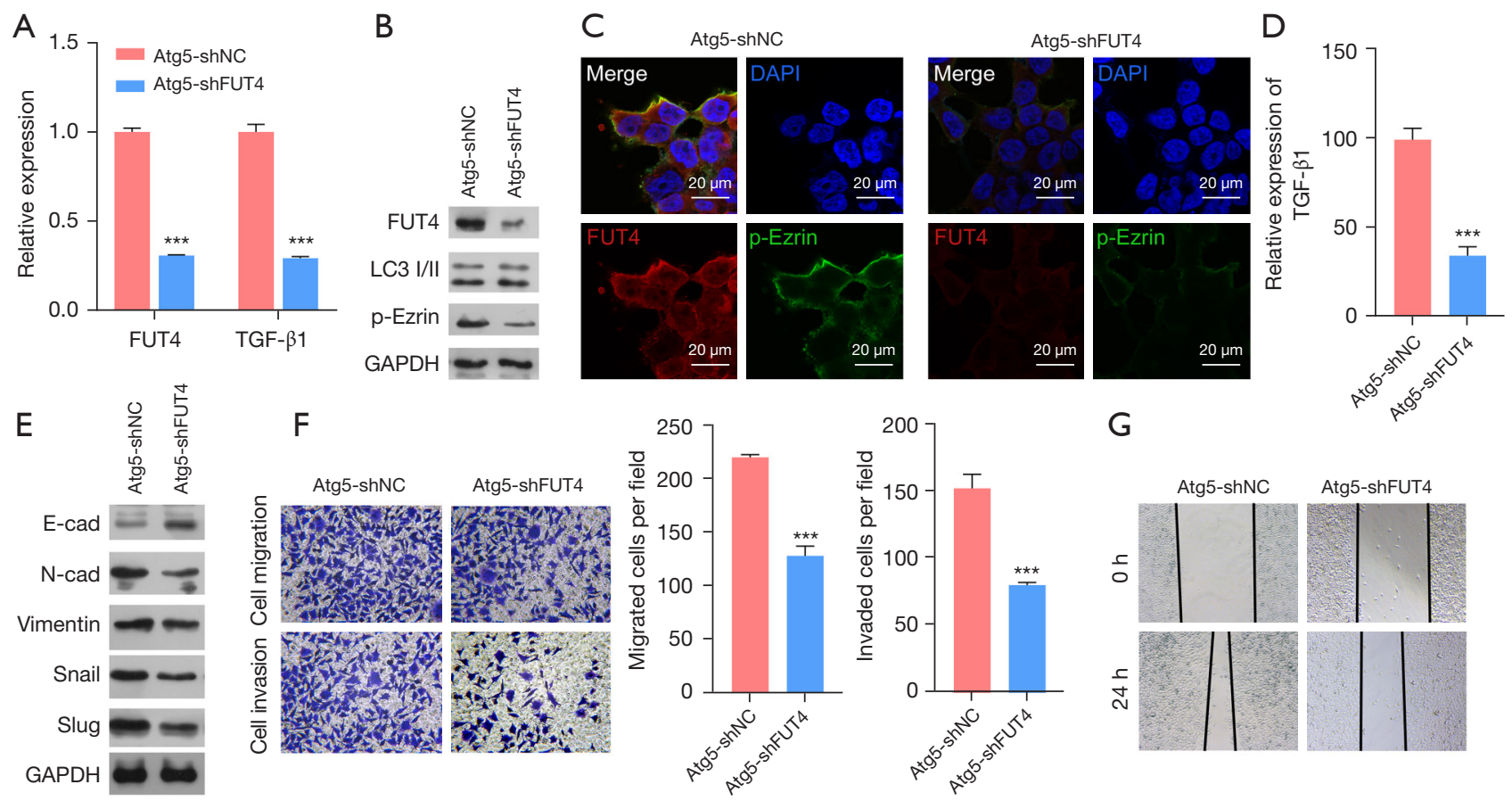

G

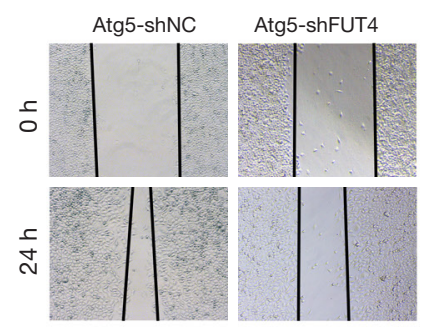

Figure 3 FUT4 silencing inhibited EMT of human lung adenocarcinoma cells induced by autophagic TAMs. (A) The relative mRNA levels of FUT4 and TGF- $\beta 1$ in ATG5-shNC-TAMs, and ATG5-shFUT4-TAMs; (B) Western blot assays showing the protein expression of FUT4, LC3, and p-Ezrin in ATG5-shNC-TAMs and ATG5-shFUT4-TAMs; (C) the expression of FUT4 and p-Ezrin in ATG5shNC-TAMs and ATG5-shFUT4-TAMs by cell immunofluorescence; (D) the expression of TGF- $\beta 1$ in ATG5-shNC-TAMs and ATG5shFUT4-TAMs by enzyme-linked immunosorbent assay; (E) ATG5-shFUT4-TAMs were stimulated with rapamycin and then cocultured with H1299 cells. The protein levels of N-cadherin (N-cad), E-cadherin (E-cad), vimentin, Snail, and Slug in the treated H1299 cells were assessed by Western blotting; (F) cell migration and invasion of the treated H1299 cells was assessed by Transwell assays ( $\times 100$ magnification), in which cells in the lower compartment were stained with crystal violet; (G) the relative wound area of the treated H1299 cells was examined by wound healing assays ( $\times 40$ magnification). ${ }^{* * *}, \mathrm{P}<0.001$ s. ATG5-shNC group. FUT4, fucosyltransferase IV; p-erzin, phosphorylated erzin; TGF- $\beta 1$, tumor growth factor $\beta 1$; TAM, tumor-associated macrophage; ATG5, autophagy related gene 5; sh, short hairpin; NC, negative control; qRT-PCR, quantitative reverse transcription polymerase chain reaction; LC3, microtubule-associated protein 1A/1B-light chain 3; GAPDH, glyceraldehyde 3-phosphate dehydrogenase.

then co-cultured with H1299 cells. The results revealed that FUT4 silencing elevated the expression of E-cad and reduced the expression of N-cad, vimentin, Snail, and Slug in H1299 cells. In addition, inhibited wound healing and cell migration and invasion rates were observed (Figure 3E-3G). These results suggested that FUT4 silencing in autophagic TAMs may suppress the phosphorylation of ezrin and TGF- $\beta 1$, which further inhibits the EMT of H1299 cells.

\section{The effects of ezrin silencing on EMT of human lung adenocarcinoma cells induced by TAM autophagy}

Since FUT4 silencing suppressed the phosphorylation of ezrin, the effects of p-ezrin expression on the EMT of H1299 cells induced by TAM autophagy was explored. Transfection of TAMs with the ezrin silencing plasmid resulted in low expression of ezrin mRNA and phosphorylated ezrin compared to control cells (Figure 4A-4C). Interestingly, ezrin silencing also inhibited the expression of TGF- $\beta 1$ (Figure $4 A, 4 D$ ). Moreover, shEzrin-TAMs were stimulated with rapamycin to induce autophagy and then co-cultured with $\mathrm{H} 1299$ cells. The results revealed that ezrin silencing elevated the expression of E-cad and reduced the expression of N-cad, vimentin, Snail, and Slug in H1299 cells (Figure 4E). Furthermore, ezrin silencing inhibited wound healing, cell migration, and cell invasion (Figure 4F,4G). These results suggested that ezrin 
A
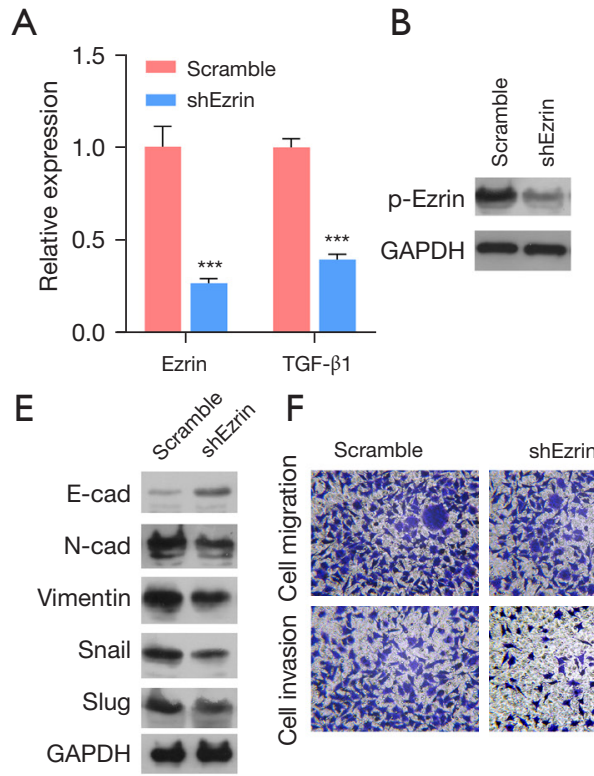

$\mathrm{F}$
C
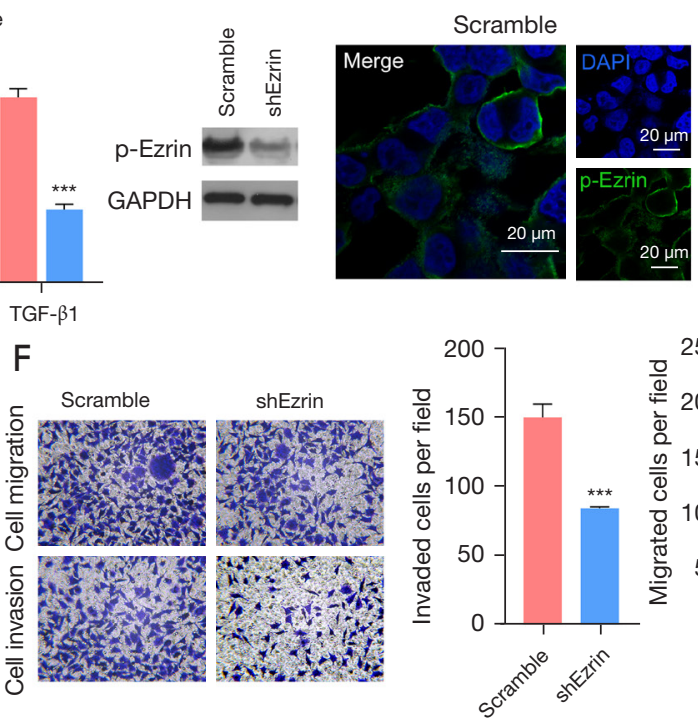
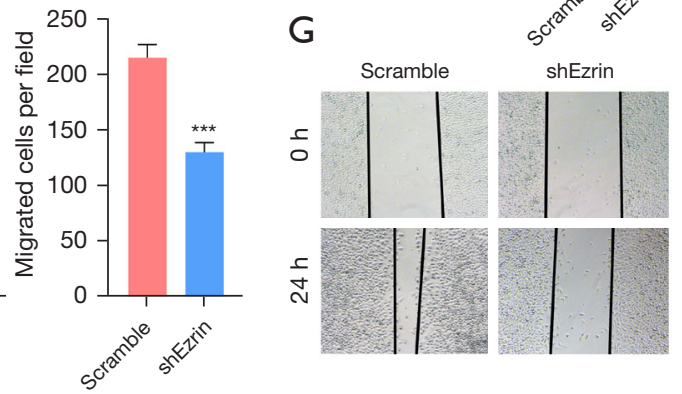

Figure 4 Ezrin silencing inhibited EMT of human lung adenocarcinoma cells. (A) The mRNA levels of ezrin and TGF- $\beta 1$ in scrambleTAMs and shEzrin-TAMs assessed by qRT-PCR; (B) the expression of p-ezrin in scramble-TAMs and shEzrin-TAMs was detected by cell immunofluorescence; (C) the expression of p-Ezrin in scramble-TAMs and shEzrin-TAMs by cell immunofluorescence; (D) the expression of TGF- $\beta 1$ in scramble-TAMs and shEzrin-TAMs was examined by enzyme-linked immunosorbent assay; (E) the shEzrin-TAMs were stimulated with rapamycin and then co-cultured with H1299 cells. The protein levels of N-cadherin (N-cad), E-cadherin (E-cad), vimentin, Snail, and Slug in the treated H1299 cells were assessed by Western blot analysis; (F) cell migration and invasion of the treated H1299 cells was examined by Transwell assays( $\times 100$ magnification), in which cells in the lower compartment were stained with crystal violet; (G) the relative wound area of the treated $\mathrm{H} 1299$ cells was determined by wound healing assays ( $\times 40$ magnification). ${ }^{* *}, \mathrm{P}<0.01$ vs. scramble group; ***, $\mathrm{P}<0.001$ vs. scramble group. TAM, tumor-associated macrophage; ATG5, autophagy related gene 5; sh, short hairpin; EMT, endothelial mesenchymal transition; GAPDH, glyceraldehyde 3-phosphate dehydrogenase.

might participate in TGF- $\beta 1$-mediated EMT of H1299 cells induced by TAM autophagy.

\section{The effects of FUT4 overexpression on EMT of human lung adenocarcinoma cells induced by autophagic TAMs}

To further verify the influence of FUT4 on the EMT of A549 cells induced by autophagic TAMs, FUT4 was over-expressed in shATG5-TAMs. Overexpression was confirmed by increased expression of FUT4 in shATG5-FUT4-TAMs compared to shATG5-NC-TAMs (Figure 5A,5B). FUT4 overexpression promoted the expression of p-ezrin and TGF- $\beta 1$, without changing the expression of LC3 (Figure $5 A-5 D$ ). Furthermore, shATG5-TAMs were stimulated with rapamycin to induce autophagy and then co-cultured with A549 cells. The results revealed that FUT4 overexpression inhibited the expression of $\mathrm{E}$-cad and increased the expression of $\mathrm{N}$-cad, vimentin, Snail, and Slug in A549 cells. Overexpression of FUT4 also promoted the wound healing ability and the cell migration and invasion rates (Figure $5 E-5 G$ ). These results suggested that FUT4 overexpression contributes to TGF- $\beta 1$-mediated EMT of A549 cells induced by of TAM autophagy.

\section{The effects of TAM autophagy on FUT4/p-ezrin expression and EMT in vivo in mice with lung adenocarcinoma}

In vivo experiments demonstrated that the tumor volumes were significantly increased in mice in the ATG5-shNCTAMs + H1299 group compared with mice in the TAMs + H1299 group. Silencing of FUT4 in the ATG5-shFUT4TAMs + H1299 group resulted in time-dependent decrease in tumor volume compared to mice in the ATG5-shNCTAMs + H1299 group (Figure 6A,6B). In addition, ATG5shNC-TAMs inhibited the tumor expression of E-cad and increased the expression of TGF- $\beta 1, \mathrm{~N}$-cad, vimentin, Snail, and Slug compared to tumors from the TAMs + 


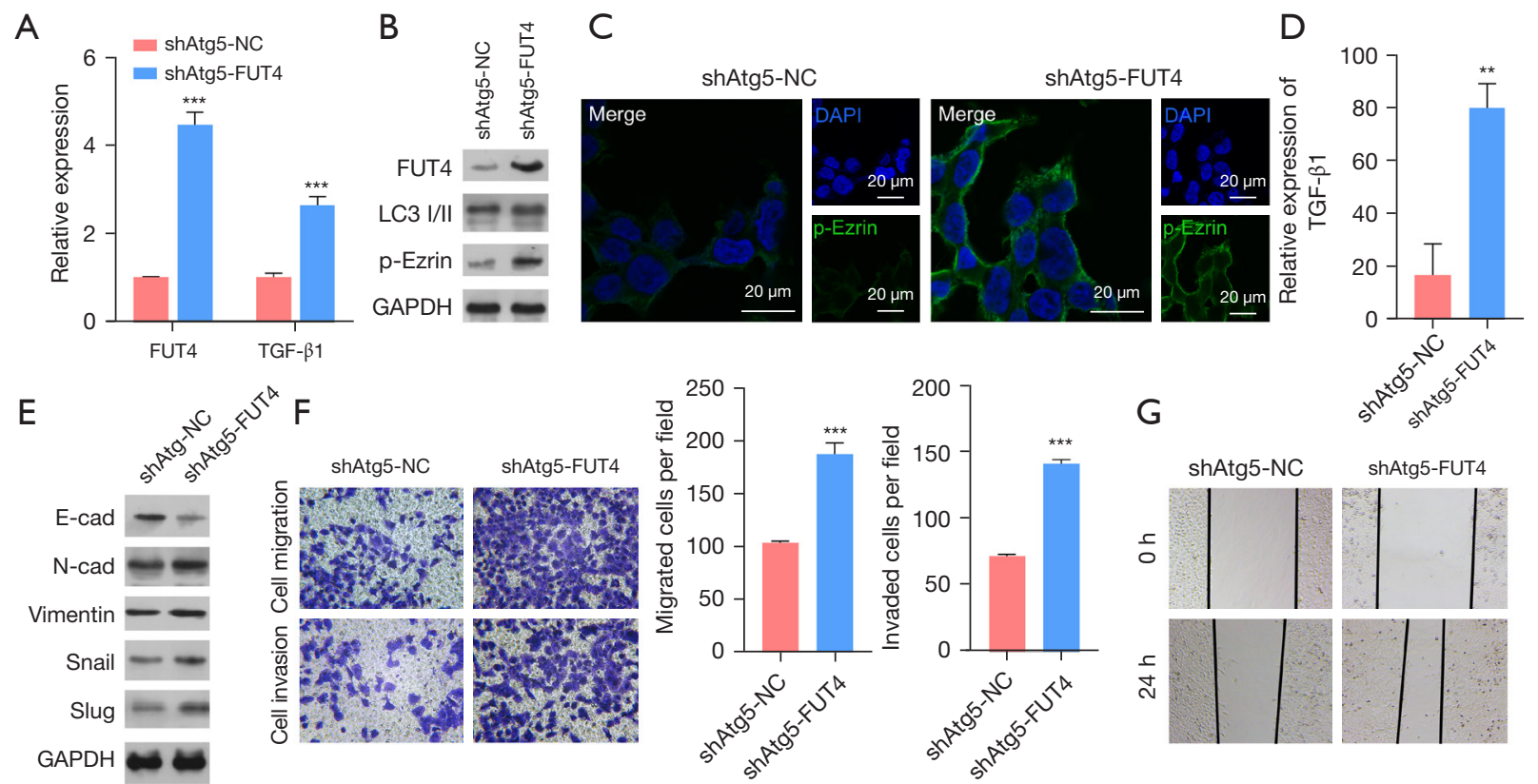

Figure 5 FUT4 overexpression promoted EMT of human lung adenocarcinoma cells induced by autophagic TAMs. (A) The relative mRNA levels of FUT4 and TGF- 31 in shATG5-NC-TAMs, and shATG5-FUT4-TAMs as determined by qRT-PCR; (B) the protein levels of FUT4, LC3, and p-ezrin in shATG5-NC-TAMs and shATG5-FUT4-TAMs were assessed by Western blotting; (C) the expression of p-ezrin in shATG5-NC-TAMs and shATG5-FUT4-TAMs was visualized by cell immunofluorescence studies; (D) the expression of TGF- $\beta 1$ in shATG5-NC-TAMs, and shATG5-FUT4-TAMs was assessed by enzyme-linked immunosorbent assays; (E) the shATG5FUT4-TAMs were stimulated with rapamycin and then co-cultured with A549 cells. The protein levels of N-cadherin (N-cad), E-cadherin (E-cad), vimentin, Snail, and Slug in the treated A549 cells were determined by Western blot analysis; (F) the cell migration and invasion of the treated A549 cells was assessed by Transwell assays ( $\times 100$ magnification), in which cells in the lower compartment were stained with crystal violet; $(\mathrm{G})$ the relative wound area of the treated A549 cells was examined by wound healing assays ( $\times 40$ magnification). **, $\mathrm{P}<0.01$ vs. shATG5-NC group; ${ }^{* * *}, \mathrm{P}<0.001$ vs. shATG5-NC group. FUT4, fucosyltransferase IV; p-erzin, phosphorylated erzin; TGF- $\beta 1$, tumor growth factor $\beta 1$; TAM, tumor-associated macrophage; ATG5, autophagy related gene 5; sh, short hairpin; NC, negative control; qRTPCR, quantitative reverse transcription polymerase chain reaction; LC3, microtubule-associated protein 1A/1B-light chain 3; GAPDH, glyceraldehyde 3-phosphate dehydrogenase.

H1299 group. Conversely, the effects of ATG5-shNCTAMs on TGF- $\beta 1$ and EMT-related proteins were reversed by ATG5-shFUT4-TAMs in lung adenocarcinoma mice (Figure 6C). These results revealed that TAM autophagy induced TGF- $\beta 1$-mediated EMT in lung adenocarcinoma mice, and this was reversed by silencing FUT4.

\section{Discussion}

Metastatic recurrence is a key factor hindering the longterm survival of patients with lung adenocarcinomas (3). The TME has been considered as a decisive factor in the development of tumors $(6,21)$ and interestingly, TAMs, which are the largest cell component in the TME, have been reported to be closely related to poor survival and prognosis in patients with solid tumors (22). Accumulating evidences have demonstrated the role of TAMs in various cancers, including activating immunosuppression, inducing tumor metastasis, promoting drug resistance to chemotherapy, and increasing tumor growth (23). Thus, TAMs may represent a potential therapeutic target for cancer treatment (8). Notably, recent studies have reported a relationship between autophagy of TAMs and tumor progression (19). However, it remains unclear whether autophagic TAMs are involved in the metastatic mechanisms of lung adenocarcinomas. The present study demonstrated that TAM autophagy induced by ATG5 increased the expression of FUT4, TGF- $\beta 1$, and p-ezrin, and promoted EMT and cell migration and invasion 


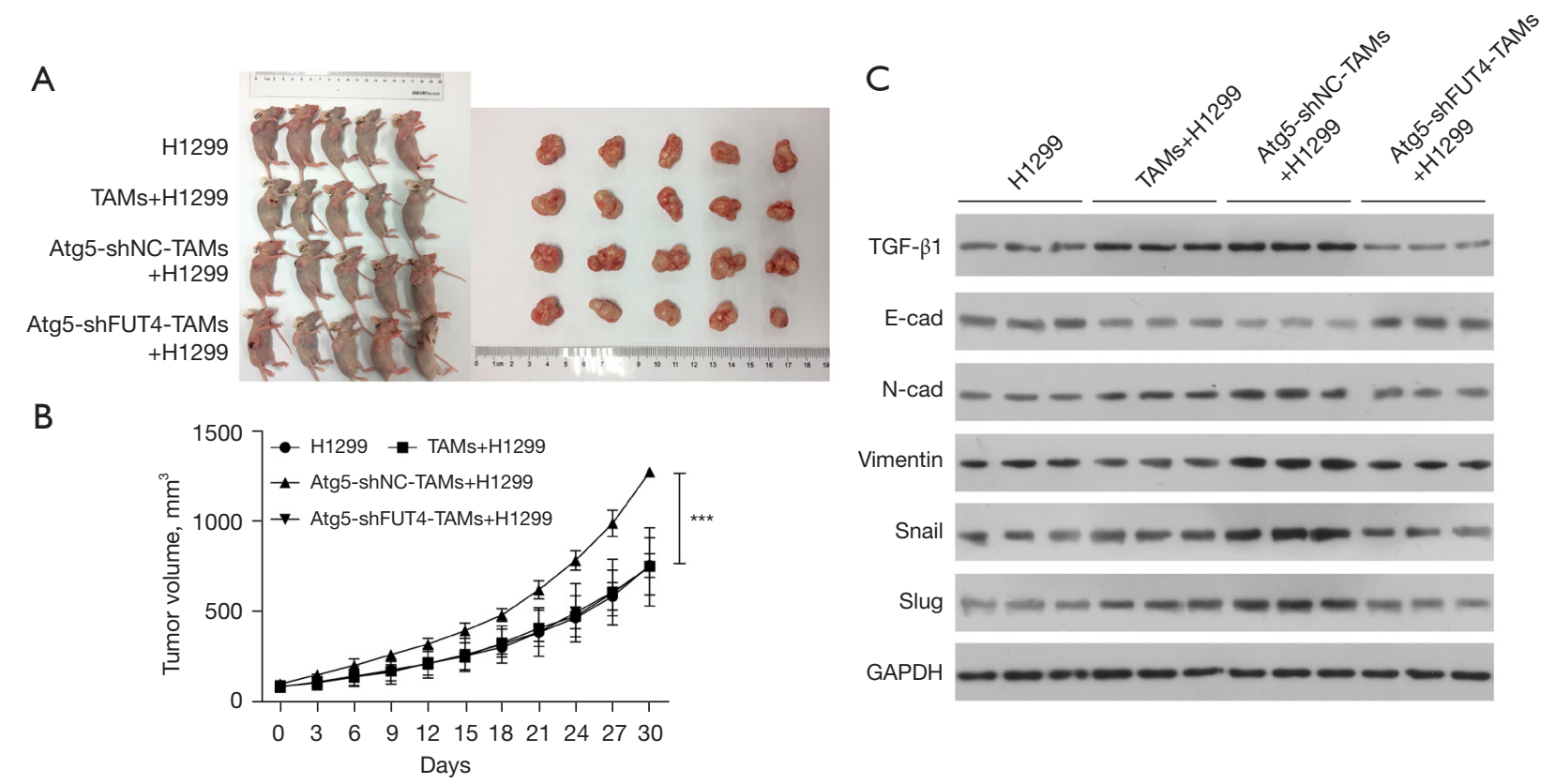

Figure 6 FUT4 was involved in EMT induced by TAM autophagy in mice with lung adenocarcinoma. (A) Representative tumors excised from tumor xenograft mice injected with H1299, TAMs + H1299, Atg5-shNC-TAMs + H1299, or Atg5-shFUT4-TAMs + H1299; (B) tumor volumes from the xenograft mice in the H1299, TAMs + H1299, Atg5-shNC-TAMs + H1299, and Atg5-shFUT4-TAMs + H1299 groups in a time-dependent manner; (C) the protein levels of TGF- $\beta 1, \mathrm{~N}$-cadherin $(\mathrm{N}$-cad), E-cadherin (E-cad), vimentin, Snail, and Slug in the excised tumors from mice in the H1299, TAMs + H1299, Atg5-shNC-TAMs + H1299, and Atg5-shFUT4-TAMs + H1299 groups were assessed by Western blot analysis. ***, $\mathrm{P}<0.001$ vs. Atg5-shNC-TAMs + H1299 group. FUT4, fucosyltransferase IV; p-erzin, phosphorylated erzin; TGF- $\beta 1$, tumor growth factor $\beta 1$; TAM, tumor-associated macrophage; ATG5, autophagy related gene 5; sh, short hairpin; NC, negative control; qRT-PCR, quantitative reverse transcription polymerase chain reaction; GAPDH, glyceraldehyde 3-phosphate dehydrogenase.

in lung adenocarcinoma cells. However, FUT4 silencing partly reversed the effects of TAM autophagy induced by ATG5. Silencing of FUT4 inhibited the expression of TGF- $\beta 1$ and p-ezrin, and suppressed EMT and cell migration and invasion in lung adenocarcinoma cells in vitro and in vivo. This suggested that the FUT4/p-ezrin pathway might participate in TGF- $\beta 1$-mediated EMT induced by TAM autophagy in lung adenocarcinoma. Numerous reports have investigated the role of autophagy in various cancers (24). Indeed, autophagy can prevent tumor initiation and progression in early tumorigenesis, but it can also contribute to the growth and aggressiveness of tumors at the later stages (24). TAMs are the main components in the TME and can participate in the metastatic processes of cancers, and autophagy can regulate the polarization of TAMs (25). This current study focused on the effects of TAM autophagy on lung adenocarcinomas.

A previous study showed that ATG5 overexpression or
mTOR deletion could induce autophagy in mouse livers with defective autophagy (26). Thus, in this study, TAM autophagy was induced by up-regulating ATG5 expression or by treatment with the mTOR inhibitor rapamycin. The results demonstrated that ATG5 overexpression induced upregulation of LC3 and beclin-1, as well as down-regulation of p62, confirming that autophagy was successfully induced in the TAMs. When the ATG5-TAMs were co-cultured with H1299 cells, the expression of E-cad was reduced and the expression of $\mathrm{N}$-cad, vimentin, Snail, and Slug were elevated. Furthermore, the cell migration and invasion ability of the H1299 cells was enhanced. EMT is a prometastasis process by which epithelial cells differentiate and acquire stromal invasiveness (27), and is characterized by the absence of E-cad and the increased expression of N-cad, vimentin, Snail, and Slug $(27,28)$. This study revealed that TAMs with autophagy promoted TGF- $\beta 1$ expression and it is well-known that TGF- $\beta 1$ stimulation 
can induce EMT (29). However, inhibiting autophagy in TAMs by down-regulating ATG5 expression exhibited the opposite effects on lung adenocarcinoma cells. Moreover, the in vivo experiments also demonstrated that autophagic TAMs increased tumor growth and EMT in mice with lung adenocarcinoma. Together, these results suggested that autophagic TAMs might promote the secretion of TGF- $\beta 1$, thereby inducing EMT to aggravate cell migration and invasion in lung adenocarcinoma cells.

Furthermore, this study investigated the possible pathways by which TAM autophagy affect the EMT process in lung adenocarcinoma. Abnormal glycosylation is a hallmark of cancers $(30,31)$. The Lewis antigen $Y$ (LEY) is an oligosaccharide bound to glycoprotein and glycolipid terminal, which is highly expressed in about $60-90 \%$ of tumors originating from the epithelium, such as kidney cancer, breast cancer, ovarian cancer, pancreatic cancer, colon cancer, and non-small cell lung cancer (31). FUT4 is a key enzyme in the oligosaccharide synthesis of LEY (32). Recent studies have reported that FUT4 drives the metastasis of lung adenocarcinomas (33), and FUT4 overexpression is accompanied by EMT in breast cancer cells (34). In the current study, autophagic TAMs increased FUT4 expression. FUT4 silencing partially reversed the effects of TAM autophagy, inhibited the expression of TGF- $\beta 1$ in TAMs, and suppressed EMT and cell migration and invasion in lung adenocarcinoma in vitro and in vivo. These results suggested that FUT4 is involved with TGF$\beta 1$-mediated EMT induced by autophagic TAMs. Notably, TAM autophagy increased the phosphorylation of ezrin, while FUT4 silencing suppressed the phosphorylation of ezrin. Furthermore, ezrin deletion in autophagic TAMs decreased TGF- $\beta 1$ expression and contributed to EMT as well as the cell migration and invasion ability of lung adenocarcinomas. Ezrin, an important regulatory protein that connects cell membrane molecules, is highly expressed in various solid tumors and is associated with poor prognosis $(35,36)$. Chen et al. reported that ezrin can mediate EMT in A549 cells through the TGF- $\beta 1$ pathway (37). The collective results indicated that TAMs with autophagy might regulate TGF- $\beta 1$-mediated EMT in lung adenocarcinoma through a FUT4-mediated ezrin phosphorylation pathway. In the present study, we cultured THP-1-derived macrophages as TAMs, which may be one of the limitations of this study. The types and the secreted cytokines are different between macrophages induced by PMA and primary infiltrated macrophages.

In conclusion, this study suggested that autophagic TAMs promote TGF- $\beta 1$ secretion through the FUT4/p-ezrin pathway and induce the EMT process, thereby facilitating tumor growth and metastasis in lung adenocarcinomas.

\section{Acknowledgments}

Funding: This research was supported by the Natural Science Research Project of Anhui Educational Committee (KJ2019A0366); the Key Project of Translational Medicine, Bengbu Medical College (BYTM2019026); the Bethune Medical Science Research Foundation (TB206006); and the Lung Cancer Precision Medicine Research Special Fund of Huilan Public Welfare-Haosen Pharmaceutical (HLHS2020-110).

\section{Footnote}

Reporting Checklist: The authors have completed the ARRIVE reporting checklist. Available at https://dx.doi. org/10.21037/jtd-21-1519

Data Sharing Statement: Available at https://dx.doi. org/10.21037/jtd-21-1519

Conflicts of Interest: Both authors have completed the ICMJE uniform disclosure form (available at https://dx.doi. org/10.21037/jtd-21-1519). The authors have no conflicts of interest to declare.

Ethical Statement: The authors are accountable for all aspects of the work in ensuring that questions related to the accuracy or integrity of any part of the work are appropriately investigated and resolved. Experiments were performed under a project license (No. 2020-146) granted by the ethics committee of the First Affiliated Hospital of Bengbu Medical College, in compliance with China national or institutional guidelines for the care and use of animals.

Open Access Statement: This is an Open Access article distributed in accordance with the Creative Commons Attribution-NonCommercial-NoDerivs 4.0 International License (CC BY-NC-ND 4.0), which permits the noncommercial replication and distribution of the article with the strict proviso that no changes or edits are made and the original work is properly cited (including links to both the formal publication through the relevant DOI and the license). See: https://creativecommons.org/licenses/by-nc-nd/4.0/. 


\section{References}

1. Bray F, Ferlay J, Soerjomataram I, et al. Global cancer statistics 2018: GLOBOCAN estimates of incidence and mortality worldwide for 36 cancers in 185 countries. CA Cancer J Clin 2018;68:394-424.

2. Hirsch FR, Scagliotti GV, Mulshine JL, et al. Lung cancer: current therapies and new targeted treatments. Lancet 2017;389:299-311.

3. Jacobsen MM, Silverstein SC, Quinn M, et al. Timeliness of access to lung cancer diagnosis and treatment: A scoping literature review. Lung Cancer 2017;112:156-64.

4. Hui M, Uppin SG, Stalin BJ, et al. Histological transformation of adenocarcinoma to small cell carcinoma lung as a rare mechanism of resistance to epidermal growth factor receptor-tyrosine kinase inhibitors: Report of a case with review of literature. Lung India 2018;35:160-3.

5. Wang JJ, Lei KF, Han F. Tumor microenvironment: recent advances in various cancer treatments. Eur Rev Med Pharmacol Sci 2018;22:3855-64.

6. Arneth B. Tumor Microenvironment. Medicina (Kaunas) 2019;56:15.

7. Emon B, Bauer J, Jain Y, et al. Biophysics of Tumor Microenvironment and Cancer Metastasis - A Mini Review. Comput Struct Biotechnol J 2018;16:279-87.

8. Zhou J, Tang Z, Gao S, et al. Tumor-Associated Macrophages: Recent Insights and Therapies. Front Oncol 2020;10:188.

9. Pathria P, Louis TL, Varner JA. Targeting TumorAssociated Macrophages in Cancer. Trends Immunol 2019;40:310-27.

10. Lin Y, Xu J, Lan H. Tumor-associated macrophages in tumor metastasis: biological roles and clinical therapeutic applications. J Hematol Oncol 2019;12:76.

11. Wang J, Li D, Cang H, et al. Crosstalk between cancer and immune cells: Role of tumor-associated macrophages in the tumor microenvironment. Cancer Med 2019;8:4709-21.

12. Liu L, Ge D, Ma L, et al. Interleukin-17 and prostaglandin E2 are involved in formation of an M2 macrophagedominant microenvironment in lung cancer. J Thorac Oncol 2012;7:1091-100.

13. Wang A, Lu C, Ning Z, et al. Tumor-associated macrophages promote Ezrin phosphorylation-mediated epithelial-mesenchymal transition in lung adenocarcinoma through FUT4/LeY up-regulation. Oncotarget 2017;8:28247-59.

14. D'Arcy MS. Cell death: a review of the major forms of apoptosis, necrosis and autophagy. Cell Biol Int 2019;43:582-92.

15. Saha S, Panigrahi DP, Patil S, et al. Autophagy in health and disease: A comprehensive review. Biomed Pharmacother 2018;104:485-95.

16. Onorati AV, Dyczynski M, Ojha R, et al. Targeting autophagy in cancer. Cancer 2018;124:3307-18.

17. Zhang L, Wang L, Guo E, et al. Silence of lncRNA CHRF protects $\mathrm{H} 9 \mathrm{c} 2$ cells against lipopolysaccharide-induced injury via up-regulating microRNA-221. Exp Mol Pathol 2019;107:43-50.

18. Zou Z, Tao T, Li H, et al. mTOR signaling pathway and mTOR inhibitors in cancer: progress and challenges. Cell Biosci 2020;10:31.

19. Chen P, Cescon M, Bonaldo P. Autophagy-mediated regulation of macrophages and its applications for cancer. Autophagy 2014;10:192-200.

20. Alonso-Nocelo M, Raimondo TM, Vining KH, et al. Matrix stiffness and tumor-associated macrophages modulate epithelial to mesenchymal transition of human adenocarcinoma cells. Biofabrication 2018;10:035004.

21. Vitale I, Manic G, Coussens LM, et al. Macrophages and Metabolism in the Tumor Microenvironment. Cell Metab 2019;30:36-50.

22. Chen Y, Song Y, Du W, et al. Tumor-associated macrophages: an accomplice in solid tumor progression. J Biomed Sci 2019;26:78.

23. Yang L, Zhang Y. Tumor-associated macrophages: from basic research to clinical application. J Hematol Oncol 2017;10:58.

24. Colhado Rodrigues BL, Lallo MA, Perez EC. The Controversial Role of Autophagy in Tumor Development: A Systematic Review. Immunol Invest 2020;49:386-96.

25. Ngabire D, Kim GD. Autophagy and Inflammatory Response in the Tumor Microenvironment. Int J Mol Sci 2017;18:2016.

26. Ni HM, Chao X, Yang H, et al. Dual Roles of Mammalian Target of Rapamycin in Regulating Liver Injury and Tumorigenesis in Autophagy-Defective Mouse Liver. Hepatology 2019;70:2142-55.

27. Pastushenko I, Blanpain C. EMT Transition States during Tumor Progression and Metastasis. Trends Cell Biol 2019;29:212-26.

28. Singh M, Yelle N, Venugopal C, et al. EMT: Mechanisms and therapeutic implications. Pharmacol Ther 2018;182:80-94.

29. Yao Y, Chen R, Wang G, et al. Exosomes derived from mesenchymal stem cells reverse EMT via TGF- $\beta 1 / \mathrm{Smad}$ 
pathway and promote repair of damaged endometrium. Stem Cell Res Ther 2019;10:225.

30. Liu HM, Ma LL, Cao B, et al. Progress in research into the role of abnormal glycosylation modification in tumor immunity. Immunol Lett 2021;229:8-17.

31. Vajaria BN, Patel PS. Glycosylation: a hallmark of cancer? Glycoconj J 2017;34:147-56.

32. Blanas A, Sahasrabudhe NM, Rodríguez E, et al. Fucosylated Antigens in Cancer: An Alliance toward Tumor Progression, Metastasis, and Resistance to Chemotherapy. Front Oncol 2018;8:39.

33. Lu HH, Lin SY, Weng RR, et al. Fucosyltransferase 4 shapes oncogenic glycoproteome to drive metastasis of lung adenocarcinoma. EBioMedicine 2020;57:102846.

34. Yang X, Liu S, Yan Q. Role of fucosyltransferase IV in epithelial-mesenchymal transition in breast cancer cells. Cell Death Dis 2013;4:e735.

35. Ko KY, Hong M, Kim TS, et al. Ezrin as a complementary marker in ocular toxicity assessment using a threedimensional reconstructed human corneal-like epithelium model, EpiOcular ${ }^{\mathrm{TM}}$. J Pharmacol Toxicol Methods 2018;92:24-33.

36. Liu Q, Xu B, Zhou W. Correlation between chemotherapy resistance in osteosarcoma patients and PAK5 and Ezrin gene expression. Oncol Lett 2018;15:879-84.

37. Chen MJ, Gao XJ, Xu LN, et al. Ezrin is required for epithelial-mesenchymal transition induced by TGF- $\beta 1$ in A549 cells. Int J Oncol 2014;45:1515-22.

(English Language Editor: J. Teoh)
Cite this article as: Wang $\mathrm{K}$, Chen $\mathrm{X}$. Autophagic tumorassociated macrophages promote the endothelial mesenchymal transition in lung adenocarcinomas through the FUT4/p-ezrin pathway. J Thorac Dis 2021;13(10):5973-5985. doi: 10.21037/ jtd-21-1519 